\title{
Combining Ability Analysis for Yield and Yield Contributing Traits in Linseed (Linum usitasissimum L.)
}

\author{
Suvarnlata D. Shivankar ${ }^{1 *}$, Beena M. Nair ${ }^{2}$, Pankaj S. Chougule ${ }^{3}$, Kshama M. Shah ${ }^{1}$, \\ Hrishikesh P. Ingole ${ }^{1}$ and Aditi S. Deshmukh ${ }^{1}$
}

${ }^{1}$ Department of Agricultural Botany, College of Agriculture, Nagpur, India

${ }^{2}$ Linseed Breeder, AICRP on Linseed and Mustard, College of Agriculture, Nagpur, India

${ }^{3}$ Post graduate Institute, MPKV, Rahuri, Ahmednagar-413722, M.S., India

*Corresponding author

\section{A B S T R A C T}

Keywords

Linseed, combining ability, GCA, SCA

Article Info

\section{Accepted:}

15 June 2021

Available Online:

10 July 2021
The present investigation was carried out with a set of ten genotypes of linseed and their forty five $\mathrm{F}_{1} \mathrm{~S}$ synthesized through diallel design excluding reciprocals. These were grown in Randomized Block Design during rabi season of 2019-2020 at farm of AICRP on linseed and mustard, College of Agriculture, Nagpur and studied for eight quantitative characters. The observations were recorded on i.e. plant height $(\mathrm{cm})$, number of branches plant ${ }^{-1}$, number of capsule plant ${ }^{-1}$, budfly infestation (\%), days to $50 \%$ flowering (on plot basis), days to maturity (on plot basis), seed yield plant ${ }^{-1}(\mathrm{~g})$ and 1000 seed weight $(\mathrm{g})$. Analysis of variance showed highly significant differences among the genotypes for all the characters studied. Four parents viz., NL 115, Neelum, Tiara and PKV NL 260 possesed positive significant gca effects and NL 115 and PKV NL 260 gaves high mean performance and were found good general combiners for seed yield plant ${ }^{-1}$, number of capsule plant ${ }^{-1}$ and 1000 seed weight and the six parents namely Neela, EC 99001, NL 367, NL 142, Padmini and NL 165 possesed negative GCA effects and were found good general combiners for bufly resistance. Hence, PKV NL 260 on crossing with EC 99001 and Neela and NL 115 on crossing with EC 99001 and Neela can be used in hybridization programme to get better transgressive segregates' for yield and budfly resistance. Among the 45 crosses studied, the crosses NL $165 \times$ Neelum, NL $142 \times$ Padmini, EC $99001 \times$ PKV NL 260 and NL $367 \times$ Tiara were found most promising as they possessed high mean performance for most the yield and yield contributing character along with negative non significant SCA effect for seed yield plant ${ }^{-1}$, number of capsule plant ${ }^{-1}$ and 1000 seed weight. The parents involved in these crosses also possessed high mean performance for yield and yield contributing characters and desirable GCA effects for yield and yield contributing characters in both the parents and thus can be used in varietal development. 


\section{Introduction}

Linseed (Linum usitatissimum L.) ia an annual self pollinated diploid $(2 x=2 n=30)$ oilseed crop belonging to linaceae family rich in oil $(41 \%)$, protein $(20 \%)$ and rich source of omega 3 fatty acids which reduces the heart diseases.

The study of combining ability helps in selection of best combiners and provides opportunity for the use of these combiners in hybridization 5 programme.

General combining ability (GCA) is primarily a function of additive gene action and additive $x$ additive gene interaction, whereas specific combining ability (SCA) is due to nonadditive gene interaction. General combining ability (GCA) and SCA effects determine the potential of parents / crosses for mobilizing them in an efficient breeding programme.

\section{Materials and Methods}

The experimental material comprised of ten parents crossed in fashion to obtain 102 crosses during rabi 2019-20. These 45 crosses along with 37 parents were grown in Randomized Block Design in two replications with the spacing of $30 \mathrm{~cm} \times 5 \mathrm{~cm}$ during rabi 2020-21 at farm of AICRP on linseed and mustard, College of Agriculture, Nagpur. Five plants were taken randomly from each plot for recording the observations.

Observations were recorded for eight quantitative characters viz., plant height $(\mathrm{cm})$, number of branches plant ${ }^{-1}$, number of capsule plant $^{-1}$, budfly infestation (\%), days to $50 \%$ flowering (on plot basis), days to maturity (on plot basis), seed yield plant ${ }^{-1}$ (g) and 1000 seed weight (g). The combining ability analysis was carried out as per standard method given by Kempthrone (1957) and ANOVA as per Panse and Sukhatme (1954).

\section{Results and Discussion}

The study of combining ability helps in selection of best combiners and provides opportunity for the use of these combiners in hybridization programme. General combining ability (GCA) is primarily a function of additive gene action and additive $\mathrm{x}$ additive gene interaction, whereas specific combining ability (SCA) is due to non-additive gene interaction. General combining ability (GCA) and SCA effects determine the potential of parents/crosses for mobilizing them in an efficient breeding programme.

The analysis of variance (Table-1) for combining ability revealed that mean square due to parents and crosses were significant for all the characters studied indicating substantial genetic variability for general combining ability among the parents and specific combining ability among the crosses. The significant mean squares for parents and crosses were also observed by Singh et al., (2016), Nirala et al., (2018) and Shekhar et al., (2019). Significance of mean squares indicates significant variation among crosses for combining ability hence allows the estimates of gca and sca effects.

\section{General Combining Ability (GCA)}

The General combining ability (GCA) effects of parent are presented in Table-2. While considering gca effect of the parent was found that none of the parent was found as a good general combiners for all the nine characters.

However NL 115, Neelum, Tiara and PKV NL 260 were found good general combiners for days to $50 \%$ flowering and days to maturity. NL 115, NL 142, Neelum and Tiaraa were found good general combiners for plant height. NL 115, Padmini, Neelum, Tiara and PKV NL 260 were found good general combiners for number of branches plant ${ }^{-1}$. 
Table.1 Analysis of variance for combining ability in linseed

\begin{tabular}{|c|c|c|c|c|c|c|c|c|c|}
\hline $\begin{array}{c}\text { Source } \\
\text { of } \\
\text { variation } \\
\end{array}$ & d.f. & $\begin{array}{l}\text { Plant } \\
\text { height }\end{array}$ & $\begin{array}{c}\text { No. of } \\
\text { branches } \\
\text { plant }^{-1}\end{array}$ & $\begin{array}{c}\text { No.of } \\
\text { capsule }^{-1} \\
\text { plant }^{-1}\end{array}$ & $\begin{array}{c}\text { Budfly } \\
\text { infestation }\end{array}$ & $\begin{array}{c}\text { Days to } \\
50 \% \\
\text { flowering }\end{array}$ & $\begin{array}{l}\text { Days to } \\
\text { maturity }\end{array}$ & $\begin{array}{l}\text { Seed yield } \\
\text { plant }^{-1}\end{array}$ & $\begin{array}{l}1000 \text { seed } \\
\text { weight }\end{array}$ \\
\hline $\begin{array}{l}\text { Due to } \\
\text { gca }\end{array}$ & 9 & $2,477.76 * *$ & $2,345.92 * *$ & $1,780.92 * *$ & $1,930.32 * *$ & $1,540.25^{* *}$ & $1,457.60 * *$ & $2,353.57 * *$ & $2,526.77 * *$ \\
\hline $\begin{array}{c}\text { Due to } \\
\text { sca }\end{array}$ & 44 & $3,309.59 * *$ & $2,656.86 * *$ & $2,560.91 * *$ & $2,310.29 * *$ & $2,332.07 * *$ & $2,480.12 * *$ & $2,479.61 * *$ & $2,926.99 * *$ \\
\hline Error & 54 & 18.394 & 14.348 & 13.298 & 10.362 & 12.851 & 13.999 & 10.601 & 25.952 \\
\hline
\end{tabular}

Table.2 General combining ability effects of the parents for different characters

\begin{tabular}{|c|c|c|c|c|c|c|c|c|c|}
\hline $\begin{array}{l}\text { Sr. } \\
\text { No. }\end{array}$ & Genotypes & $\begin{array}{c}\text { Plant } \\
\text { height } \\
(\mathrm{cm})\end{array}$ & $\begin{array}{c}\text { Number of } \\
\text { branches } \\
\text { plant }^{-1}\end{array}$ & $\begin{array}{c}\text { Number of } \\
\text { capsules }^{-1} \\
\text { plant }^{-1}\end{array}$ & $\begin{array}{c}\text { Bud fly } \\
\text { infestation } \\
(\%)\end{array}$ & $\begin{array}{c}\text { Days to } \\
\mathbf{5 0 \%} \\
\text { flowering }\end{array}$ & $\begin{array}{c}\text { Days } \\
\text { to } \\
\text { maturity }\end{array}$ & $\begin{array}{l}\text { Seed yield } \\
\text { plant }^{-1} \\
\text { (g) }\end{array}$ & $\begin{array}{l}1000 \text { seed } \\
\text { weight } \\
\text { (g) }\end{array}$ \\
\hline & Lines & & & & & & & & \\
\hline 1 & NL 115 & $5.664 * *$ & $2.666 * *$ & $10.049 * *$ & $4.371 * *$ & $3.951 * *$ & $7.256^{* *}$ & $5.694 * *$ & $5.179 * *$ \\
\hline 2 & NL 165 & -2.272 & 0.26 & -0.147 & -3.65 & -0.809 & -1.079 & -0.257 & $2.323^{*}$ \\
\hline 3 & NL 142 & $4.332 * *$ & -5.637 & -2.187 & -3.377 & -6.829 & -3.457 & -10.252 & -5.088 \\
\hline 4 & NL 367 & -10.598 & -8.007 & -6.98 & -4.855 & -7.371 & -6.716 & -6.304 & -7.888 \\
\hline 5 & Padmini & -1.347 & $8.385^{* *}$ & -0.899 & -2.055 & -2.078 & -2.125 & -0.832 & $5.948 * *$ \\
\hline 6 & Neelum & $13.689 * *$ & $18.267 * *$ & $11.93 * *$ & $15.829 * *$ & $15.196 * *$ & $12.566 * *$ & $18.939 * *$ & $19.141 * *$ \\
\hline 7 & Neela & -10.745 & -10.617 & -12.736 & -9.975 & -7.18 & -8.612 & -10.017 & -13.192 \\
\hline 8 & Tiara & $15.183 * *$ & $5.799 * *$ & $8.081 * *$ & $7.974 * *$ & $7.515 * *$ & $9.003 * *$ & $3.822 * *$ & $3.335 * *$ \\
\hline 9 & EC 99001 & -14.983 & -14.628 & -11.217 & -12.844 & -8.754 & -10.3 & -11.135 & -14.695 \\
\hline 10 & $\begin{array}{c}\text { PKV NL } \\
260\end{array}$ & 1.077 & $3.513 * *$ & $4.106 * *$ & $8.581 * *$ & $6.36 * *$ & $3.465 * *$ & $10.341 * *$ & $4.936 * *$ \\
\hline
\end{tabular}


Table.3 Specific combining ability effects of crosses for different characters

\begin{tabular}{|c|c|c|c|c|c|c|c|c|c|}
\hline $\begin{array}{l}\text { Sr. } \\
\text { No. }\end{array}$ & Genotypes & $\begin{array}{c}\text { Plant } \\
\text { height } \\
(\mathrm{cm})\end{array}$ & $\begin{array}{c}\text { Number } \\
\text { of } \\
\text { branches } \\
\text { plant }^{-1}\end{array}$ & $\begin{array}{l}\text { Number } \\
\text { of } \\
\text { capsules } \\
\text { plant }^{-1}\end{array}$ & $\begin{array}{c}\text { Bud fly } \\
\text { infestation } \\
(\%)\end{array}$ & $\begin{array}{c}\text { Days to } \\
50 \% \\
\text { Flowering }\end{array}$ & $\begin{array}{c}\text { Days } \\
\text { to } \\
\text { maturity }\end{array}$ & $\begin{array}{c}\text { Seed } \\
\text { yield plant }{ }^{-1} \\
(\mathrm{~g})\end{array}$ & $\begin{array}{c}1000 \\
\text { seed } \\
\text { weight }(g)\end{array}$ \\
\hline & Lines & & & & & & & & \\
\hline 1 & NL $115 \times$ NL 165 & -32.94 & -29.51 & -34.69 & -27.82 & -28.61 & -31.92 & -32.88 & -37.36 \\
\hline 2 & NL $115 \times$ NL 142 & $55.66 * *$ & $42.64 * *$ & $41.36^{* *}$ & $51.76 * *$ & $34.96 * *$ & $76.12 * *$ & $25.91 * *$ & $52.88 * *$ \\
\hline 3 & NL $115 \times$ NL 367 & 2.67 & -2.66 & -7.55 & -9.98 & 2.47 & -12.45 & -10.98 & -8.77 \\
\hline 4 & NL $115 \times$ Padmini & -29.46 & -8.43 & -22.58 & -11.22 & -19.75 & -21.41 & -27.73 & -33.33 \\
\hline 5 & NL $115 \times$ Neelum & -49.74 & -49.22 & -49.95 & -45.79 & -45.31 & -45.75 & -51.59 & -53.27 \\
\hline 6 & NL $115 \times$ Neela & $19.14 * *$ & $44.45 * *$ & $29.81 * *$ & $32.91 * *$ & $31.01 * *$ & $31.24 * *$ & $45.48 * *$ & $33.65 * *$ \\
\hline 7 & NL $115 \times$ tiara & $49.21 * *$ & $60.54 * *$ & $60.49 * *$ & $65.96 * *$ & $64.82 * *$ & $49.12 * *$ & $61.64 * *$ & $54.63 * *$ \\
\hline 8 & $\begin{array}{c}\text { NL } 115 \times \text { EC } \\
99001\end{array}$ & -19.90 & -15.81 & -25.88 & -17.19 & -22.22 & -22.25 & -21.19 & -17.48 \\
\hline 9 & $\begin{array}{c}\text { NL } 115 \times \text { PKV NL } \\
260\end{array}$ & -38.50 & -37.59 & -45.84 & -40.95 & -37.88 & -35.68 & -45.80 & -40.01 \\
\hline 10 & NL $165 \times$ NL142 & 5.12 & $9.67 * *$ & 13.25 & $11.73 * *$ & $11.30 * *$ & -0.58 & $22.67 * *$ & $22.49 * *$ \\
\hline 11 & NL $165 \times$ NL 367 & -16.70 & -20.04 & -21.13 & -17.89 & -19.33 & -18.58 & -20.91 & -24.21 \\
\hline 12 & NL $165 \times$ Padmini & $67.13 * *$ & $49.07 * *$ & $48.13 * *$ & $14.48 * *$ & $30.59 * *$ & $14.58 * *$ & $29.12 * *$ & $81.74 * *$ \\
\hline 13 & NL $165 \times$ Neelum & -20.65 & -32.82 & -23.31 & -24.01 & -25.93 & -1.67 & -26.37 & -34.59 \\
\hline 14 & NL $165 \times$ Neela & -11.39 & -1.52 & -8.76 & -1.29 & -7.16 & -1.19 & $13.31 * *$ & -6.29 \\
\hline 15 & NL $165 \times$ Tiara & -43.53 & -34.16 & -36.57 & -29.82 & -32.85 & -33.37 & -29.10 & -34.81 \\
\hline 16 & NL 165×EC99001 & $40.81 * *$ & $41.87 * *$ & $47.98 * *$ & $42.80 * *$ & $40.35 * *$ & $42.76 * *$ & $40.05 * *$ & $37.01 * *$ \\
\hline 17 & NL $165 \times$ NL260 & $59.75 * *$ & $68.73 * *$ & $69.16 * *$ & $67.87 * *$ & $74.23 * *$ & $72.49 * *$ & $55.07 * *$ & $58.38 * *$ \\
\hline 18 & NL $142 \times$ NL 367 & -26.12 & -17.68 & -20.27 & -20.91 & -14.18 & -16.76 & -11.46 & -18.27 \\
\hline 19 & NL $142 \times$ Padmini & -31.75 & -28.12 & -23.27 & -20.04 & -15.01 & -18.04 & -14.61 & -29.31 \\
\hline 20 & NL $142 \times$ Neelum & 5.41 & $17.17 * *$ & $15.87 * *$ & $11.15 * *$ & -2.28 & -14.10 & $7.47 * *$ & $8.79 *$ \\
\hline 21 & NL 142×Neela & -24.28 & -11.90 & -13.79 & -12.18 & -15.01 & -14.93 & -5.95 & -9.08 \\
\hline 22 & NL $142 \times$ tiara & $124.10 * *$ & $35.44 * *$ & $39.71 * *$ & $29.57 * *$ & $27.57 * *$ & $25.38 * *$ & -2.91 & $18.49 * *$ \\
\hline 23 & NL 142×EC99001 & -2.72 & 1.76 & $8.12 * *$ & $4.70^{*}$ & $14.39 * *$ & $11.84 * *$ & $18.86 * *$ & $8.17 *$ \\
\hline 24 & NL & 4.58 & $10.87 * *$ & $15.74 * *$ & $17.38 * *$ & -2.45 & -13.77 & $24.74 * *$ & $19.79 * *$ \\
\hline
\end{tabular}




\begin{tabular}{|c|c|c|c|c|c|c|c|c|c|}
\hline & 142×PKVNL260 & & & & & & & & \\
\hline 25 & NL $367 \times$ Padmini & $38.50 * *$ & $32.62 * *$ & $46.00 * *$ & $52.21 * *$ & $36.24 * *$ & $45.72 * *$ & $43.29 * *$ & $36.08 * *$ \\
\hline 26 & NL 367×Neelum & $63.47 * *$ & $63.24 * *$ & $61.67 * *$ & $66.33 * *$ & $66.96 * *$ & $74.03 * *$ & $50.52 * *$ & $63.89 * *$ \\
\hline 27 & NL367×Neela & -8.24 & -9.23 & -8.00 & -11.94 & -10.47 & -10.44 & -11.59 & -6.37 \\
\hline 28 & NL $367 \times$ Tiara & -37.19 & -27.92 & -30.44 & -32.06 & -28.77 & -31.33 & -26.59 & -26.18 \\
\hline 29 & $\begin{array}{l}\text { NL 367x } \\
\text { EC99001 }\end{array}$ & -2.61 & -3.41 & -6.46 & -7.55 & -7.84 & -6.38 & -8.23 & -7.58 \\
\hline 30 & $\begin{array}{c}\text { NL 367× } \\
\text { PKVNL260 }\end{array}$ & 4.58 & $10.87 * *$ & $15.74 * *$ & $17.38 * *$ & -2.45 & -0.04 & $24.74 * *$ & $19.79 * *$ \\
\hline 31 & Padmini $\times$ Neelum & $11.71 * *$ & $66.72 * *$ & $13.18 * *$ & $7.75 * *$ & $30.39 * *$ & $10.02 * *$ & $40.76 * *$ & $69.08 * *$ \\
\hline 32 & Padmini $\times$ Neela & $9.42 * *$ & $10.99 * *$ & -2.16 & $10.05 * *$ & 2.88 & $7.21 * *$ & 1.84 & -4.34 \\
\hline 33 & Padminix Tiara & -23.99 & -32.99 & -28.87 & -20.71 & -27.92 & -18.41 & -21.72 & -32.13 \\
\hline 34 & $\begin{array}{l}\text { Padminix } \\
\text { EC99001 }\end{array}$ & -13.89 & -23.01 & -15.68 & -11.29 & -16.06 & -13.32 & -15.49 & -20.28 \\
\hline 35 & $\begin{array}{c}\text { Padminix } \\
\text { PKVNL260 }\end{array}$ & $31.33 * *$ & $23.10 * *$ & $35.91 * *$ & $23.28 * *$ & $24.00 * *$ & $37.54 * *$ & $16.15^{* *}$ & $16.75 * *$ \\
\hline 36 & Neelum $\times$ Neela & -33.18 & -34.54 & -25.30 & -29.70 & -33.01 & -29.70 & -35.90 & -34.66 \\
\hline 37 & Neelum $\times$ Tiara & -62.67 & -53.59 & -49.74 & -53.15 & -50.52 & -51.14 & -53.92 & -53.27 \\
\hline 38 & $\begin{array}{l}\text { Neelum } \times \\
\text { EC99001 }\end{array}$ & -26.25 & -31.39 & -25.52 & -30.46 & -31.73 & -28.41 & -34.24 & -32.83 \\
\hline 39 & $\begin{array}{c}\text { Neelum× } \\
\text { PKVNL260 }\end{array}$ & $16.54 * *$ & -17.53 & -12.42 & $15.59 * *$ & $19.64 * *$ & -10.78 & $54.72 * *$ & 5.16 \\
\hline 40 & Neela $\times$ Tiara & $49.05 * *$ & $23.10 * *$ & $35.36 * *$ & $30.60 * *$ & $35.25 * *$ & $53.49 * *$ & $25.55 * *$ & $34.50 * *$ \\
\hline 41 & Neela× EC99001 & $23.69 * *$ & $5.24^{*}$ & $9.02 * *$ & $6.00 * *$ & $27.99 * *$ & 2.22 & $5.93 * *$ & $13.13 * *$ \\
\hline 42 & $\begin{array}{c}\text { Neelax } \\
\text { PKVNL260 }\end{array}$ & -10.13 & -11.32 & -13.36 & -15.15 & -21.99 & -16.35 & -24.85 & -16.57 \\
\hline 43 & Tiara× EC99001 & $21.36 * *$ & $44.33 * *$ & $37.25 * *$ & $36.17 * *$ & $34.02 * *$ & $43.18 * *$ & $51.47 * *$ & $39.00 * *$ \\
\hline 44 & $\begin{array}{c}\text { Tiarax } \\
\text { PKVNL260 }\end{array}$ & $44.80 * *$ & $65.19 * *$ & $60.43 * *$ & $57.75 * *$ & $62.91 * *$ & $52.41 * *$ & $65.99 * *$ & $71.87 * *$ \\
\hline 45 & $\begin{array}{c}\text { EC99001× } \\
\text { PKVNL260 }\end{array}$ & -18.44 & -20.27 & -22.19 & -25.12 & -24.97 & -19.54 & -27.86 & -21.98 \\
\hline 46 & $\operatorname{SE}\left(s_{\mathrm{ij}}\right)$ & 2.794 & 2.467 & 2.375 & 2.097 & 2.335 & 2.437 & 2.121 & 3.318 \\
\hline
\end{tabular}


NL 115, Neelum, Tiara and PKV NL 260 were found good general combiners for number of capsule plant ${ }^{-1}$, seed yield plant $^{-1}$, 1000 seed weight. NL 115, Neelum, Tiara and PKV NL 260 were found good general combiners for budfly resistance in linseed. These parents showed both additive and non additive type of gene action involving different combinations of high and low general combiners. Similar result was also reported by (Moneum et al., 2014 and Singh et al., 2016).

\section{Specific Combining Ability (SCA)}

The choice of parents for hybridization influences the success in any crop improvement program. The selection of parents based on per se performance is not always good indicator of superior combining parents. Hence, the combining ability analysis serve as an important tool for selection of parents with highest breeding value. The parents with high general combining ability effects may be used for improvement of individual trait per se. The SCA effects do not contribute tangibly in the improvement of self fertilizing crops, except where commercial exploitation of heterosis is feasible. The SCA value represents the dominance and epistatic interactions which are non-fixable in nature and related to heterosis (Griffing, 1956). Therefore, if both or one of the parents involved in the crosses with high SCA values they could be successfully exploited in varietal improvement program and expected to give superior transgressive segregants. Among the 45 crosses studied, the crosses NL $165 \times$ Neelum, NL $142 \times$ Padmini, EC $99001 \times$ PKV NL 260 and NL $367 \times$ Tiara were found most promising as they possessed high mean performance for most the yield and yield contributing character along with negative non significant SCA effect for seed yield plant $^{-1}$, number of capsule plant ${ }^{-1}$ and 1000 seed weight. The parents involved in these crosses also possessed high mean performance for yield and yield contributing characters and desirable GCA effects for yield and yield contributing characters in both the parents. The above findings less more closely in agreement with the result of earlier reports (Nirala et al., 2018 and Shekhar et al., 2019).

Based on the above discussion combining ability analysis revealed that NL 115, Neelum, Tiara and PKV NL 260 were found good general combiners for yield and yield contributing characters. The crosses NL $165 \times$ Neelum, EC $99001 \times$ PKV NL 260, NL $367 \times$ Tiara and NL $142 \times$ Padmini were identified most promising crosses for yield and its components based on sca effects.

\section{References}

Moneam Abdel, M. A. 2014. Diallel cross analysis for yield and its related traits in some genotypes of flax (Linum usitatissimum L.). Int. J. plant breeding and genetics 8 (3): 153-163.

Nirala, R., N., Rani, S. Acharya, R. Vishwakarma, T. Ranjan, B. Prasad and A. Pal. 2018. Combining ability analysis for grain yield and its component traits in linseed (Linum usitatissimum L.). Curr. J. App. Sci. Tec. 31(4): 1-12.

Shekhar, R., Pratap, N., Singh, R. P., Singh, A., Chauhan, M. P. and Vishnoi, R. K. 2019.Combing ability analysis in Linseed (Linum ussitatissimum L.). JPP; SP 3: $22-25$.

Singh, N., Chandrawati, R. Kumar, S. Kumar and H. K. Yadav. 2016. Study on genetic combining ability estimates for yield and related traits in (Linum usitatissimum L). AJCS 10(11):15941600

Singh, V. K., V. Sharma, V., M. Chaudhary, S. K. Paswan, A. Ahmad, M. Verma and M. P. Chauhan. 2016. Combining 
ability analysis in Linseed (Linum usitatissimum L) for improvement of seed yield and its component traits. J. Appl. Nat. Sci. 8(1): 1-4.

\section{How to cite this article:}

Suvarnlata D. Shivankar, Beena M. Nair, Pankaj S. Chougule, Kshama M. Shah, Hrishikesh P. Ingole and Aditi S. Deshmukh. 2021. Combining Ability Analysis for Yield and Yield Contributing Traits in Linseed (Linum usitasissimum L.). Int.J.Curr.Microbiol.App.Sci. 10(07): 386-392. doi: https://doi.org/10.20546/ijcmas.2021.1007.042 